\title{
Myocardial perfusion imaging in cardiac sarcoidosis: A "sine qua non" for prognosis assessment?
}

\author{
Georgios Benetos, MD, PhD, a and Andreas A. Giannopoulos, MD, PhD ${ }^{\mathrm{a}, \mathrm{b}}$ \\ a Cardiac Imaging, Department of Nuclear Medicine, University Hospital Zurich, Zurich, Switzerland \\ b Cardiology Department, University Hospital Zurich, Zurich, Switzerland
}

Received Nov 10, 2019; accepted Nov 11, 2019

doi: 10.1007/s12350-019-01964-w

\section{See related article pp. 1745-1756}

Sarcoidosis is a heterogeneous, granulomatous disorder of unknown etiology with multiorgan involvement. Cardiac involvement may range from asymptomatic granuloma infiltration to clinically conduction disturbances, ventricular arrhythmias, progressive heart failure and sudden death and has important prognostic and therapeutic implications. Multimodality imaging plays an important role in the diagnosis of cardiac sarcoidosis but also in the assessment of treatment efficacy. To date gallium-67 scintigraphy, cardiac magnetic resonance imaging (cMRI), and ${ }^{18}$ F-Fluorodeoxyglucose positron emission tomography (FDG-PET) have been included as imaging diagnostic tests in guidelines and in expert consensus documents. ${ }^{1,2}$ FDG-PET and increasingly cMRI aside diagnostic purposes can also provide information for the risk assessment and management of the disease. ${ }^{3}$

On a pathophysiologic basis, cardiac sarcoidosis may alter coronary microcirculation leading to myocardial perfusion defects. These defects, however, may represent either active inflammation or scar, with abnormal FDG uptake representing inflammation. Based on the different uptake patterns, various classifications of the scintigraphic images (FDG-PET and myocardial perfusion imaging-MPI) have been proposed: early

Reprint requests: Andreas A. Giannopoulos, MD, PhD, Cardiac Imaging, Department of Nuclear Medicine, University Hospital Zurich, Raemistrasse 100, 8091 Zurich, Switzerland; andreas.giannopoulos@usz.ch

J Nucl Cardiol 2021;28:1757-9.

$1071-3581 / \$ 34.00$

Copyright (C) 2019 American Society of Nuclear Cardiology. (only FDG-positive), progressive inflammatory (FDGpositive without major perfusion defects), peak active (high SUV FDG uptake with small perfusion defects), progressive myocardial impairment (high SUV FDG uptake with large perfusion defects) or fibrosis-predominant (FDG negative, but with perfusion defects). ${ }^{4}$ Another staging system resembles the staging of pulmonary sarcoidosis: Stage 0 (normal FDG, normal perfusion), Stage 1 (FDG-positive, normal perfusion), Stage 2 (FDG-positive with perfusion defects in the same myocardial territory), Stage 3 (FDG-positive with perfusion defects in different territories) or Stage 4 (normal FDG, but with perfusion defects). ${ }^{5}$ Lastly, a three-category scheme has been reported with prognostic implications: (1) normal perfusion and metabolism, (2) abnormal perfusion or metabolism, and (3) abnormal perfusion and metabolism. ${ }^{6}$ Nevertheless, the change in the above patterns following immunosuppressive therapy has not been extensively investigated.

In the present issue of $\mathrm{JNC}$, Koyanagawa et $\mathrm{al}^{7}$ retrospectively evaluated myocardial perfusion changes in response to immunosuppressive therapy and their potential prognostic significance in patients with cardiac sarcoidosis. Thirty-eight patients with cardiac sarcoidosis who had undergone both FDG-PET and 99mTc Single Photon Emission Tomography (SPECT-MPI) prior and post-steroid therapy were included. The FDG uptake pattern was described as focal or focal on diffuse and was quantified through the evaluation of cardiac metabolic volume (CMV) and activity (CMA). ${ }^{4,5}$ The summed rest score of baseline and follow-up rest MPI was compared automatically and, accordingly, patients were classified into recovery and non-recovery group. The difference in the incidence of MACE was assessed, including all-cause death, supraventricular or ventricular arrhythmia, new atrioventricular block and hospitalization. During a median follow-up of close to 3 years, patients in the recovery group were shown to have 
suffered less MACE, an effect primarily driven by the event rate of arrhythmias. In contrast, there was no difference in the rate of death, atrioventricular block or hospitalization between the two groups. Interestingly, the rate of MACE was independent from reduced ejection fraction $(<50 \%)$ at baseline. Moreover, in the present study, inflammation intensity was significantly reduced in both groups, with no statistically significant difference in any quantitative marker of inflammation (SUVmax, CMV, CMA) post-steroid therapy.

Although underpowered to detect the level of potential differences in MACE, the elegant study by Koyanagawa et al approached an important issue, evaluating the potential incremental value of sequential MPI for prognosis assessment in cardiac sarcoidosis. It remains elusive whether, following coronary artery disease exclusion, the presence of MPI defect in cardiac sarcoidosis corresponds to a dysfunction of microcirculation or to a permanent scar. To date, the interest in prognosis assessment in cardiac sarcoidosis through FDG-PET has been focused in the quantification of myocardial inflammatory activity both at baseline and at follow-up. Indeed, it has been shown that the decrease in inflammatory activity in sequential examinations is associated with an improvement in ejection fraction and lower number of events. ${ }^{8,9}$ Various markers have been proposed and validated, including SUVmax, CMA, and CMV, also used in the present study. ${ }^{10,11}$ However, reliable quantification of inflammation in cardiac sarcoidosis, through a hotspot imaging modality such as FDG-PET, poses significant technical and methodological challenges. ${ }^{12}$ These include the need for electrocardiographic gating in an FDG-PET examination (not available in all scanners), the lack of standardization in patient preparation, and difficulty in suppression of myocardial FDG uptake, especially in the presence of hibernating myocardium.

The idea of incorporating information from MPI in prognostic assessment of patients with cardiac sarcoidosis seems appealing for nuclear cardiologists given the fact that they are familiarized with the evaluation of defects in the cold spot imaging of MPI, whose quantification is more standardized. The prognostic significance of this blending of information has already been demonstrated in two recent retrospective studies with a larger pool of patients. ${ }^{6,13}$ More specifically, both studies showed the prognostic role of the mismatch pattern (abnormal perfusion and metabolism). However, none evaluated the temporal changes in perfusion as in the present study.

In most of the studies investigating prognostic factors in cardiac sarcoidosis, the majority of events refer to ventricular arrhythmias. Thus, it is reasonable to presume that in patients responding to immunosuppressive therapy, a reduction in inflammatory activity together with an improvement in myocardial perfusion and perhaps in ejection fraction substantially reduces the ventricular arrhythmia risk. Further defining risk assessment in cardiac sarcoidosis, several other markers have shown prognostic significance. The presence of focal FDG uptake in right ventricle in PET imaging has been associated with high event rate. ${ }^{6}$ This marker, also, does not mandate quantification, but despite its high specificity, has a low sensitivity. Moreover, recent advances in hybrid FDG/magnetic resonance imaging (MRI) show promise in prognostic evaluation of cardiac sarcoidosis. ${ }^{14}$ Indeed, in a recent study, concomitant abnormalities in both PET (as expressed by focal FDG uptake) and in MRI (as expressed by late gadolinium enhancement) were the strongest predictive factors for events, even after adjusting for ejection fraction. ${ }^{15}$ This finding, obviously, is in accordance with the mismatch pattern of hybrid PET/CT imaging. Unfortunately, as a significant percentage of these patients undergo implantation of defibrillator, sequential imaging with MRI is not always an option.

Before reaching conclusions for the most reliable prognostic factors by imaging in cardiac sarcoidosis the following points must be taken into consideration:

1. The majority of studies investigating prognosis in this patient group are retrospective in design, and patients with either mid-range or preserved ejection fraction are included. The category of patients with heart failure with reduced ejection fraction, possibly extended scar or fibrosis, and potentially absence of inflammation is sub-optimally represented.

2. Acquisition protocols, immunosuppressive therapies, and timing intervals between repeated imaging may vary between studies and are not standardized.

3. No study to date has provided robust prognostic information, which would allow perhaps the avoidance of implantation of a defibrillator.

In conclusion, the fusion of information derived from sequential FDG-PET and MPI potentially supports the best predictive model both in monitoring of therapy but also for prognosis assessment for patients with cardiac sarcoidosis. Nevertheless, large prospective studies with predefined cutoff points, acquisition, and management protocols are mandated to confirm this theory.

\section{Disclosure}

The University Hospital Zurich holds a research contract with GE Healthcare. Dr. Giannopoulos reports a research Grant from the Iten-Kohaut-Foundation. 


\section{References}

1. Birnie DH, Sauer WH, Bogun F, Cooper JM, Culver DA, Duvernoy CS, et al. HRS expert consensus statement on the diagnosis and management of arrhythmias associated with cardiac sarcoidosis. Heart Rhythm 2014;11:1305-23.

2. Ishida Y, Yoshinaga K, Miyagawa M, Moroi M, Kondoh C, Kiso $\mathrm{K}$, et al. Recommendations for (18)F-fluorodeoxyglucose positron emission tomography imaging for cardiac sarcoidosis: Japanese Society of Nuclear Cardiology recommendations. Ann Nucl Med 2014;28:393-403.

3. Writing Group, Document reading Group, EACVI Reviewers: This document was reviewed by members of the EACVI Scientific Documents Committee for 2014-2016 and 2016-2018. A joint procedural position statement on imaging in cardiac sarcoidosis: from the Cardiovascular and Inflammation \& Infection Committees of the European Association of Nuclear Medicine, the European Association of Cardiovascular Imaging, and the American Society of Nuclear Cardiology. Eur Heart J Cardiovasc Imaging 2017;18:1073-89.

4. Okumura $\mathrm{W}$, Iwasaki $\mathrm{T}$, Toyama $\mathrm{T}$, Iso $\mathrm{T}$, Arai $\mathrm{M}$, Oriuchi $\mathrm{N}$, et al. Usefulness of fasting 18F-FDG PET in identification of cardiac sarcoidosis. J Nucl Med 2004;45:1989-98.

5. Berman JS, Govender P, Ruberg FL, Mazzini M, Miller EJ. Scadding revisited: A proposed staging system for cardiac sarcoidosis. Sarcoidosis Vasc Diffuse Lung Dis 2014;31:2-5.

6. Blankstein R, Osborne M, Naya M, Waller A, Kim CK, Murthy $\mathrm{VL}$, et al. Cardiac positron emission tomography enhances prognostic assessments of patients with suspected cardiac sarcoidosis. J Am Coll Cardiol 2014;63:329-36.

7. Koyanagawa K, Naya M, Aikawa T, Manabe O, Furuya S, Kuzume M, et al. The rate of myocardial perfusion recovery after steroid therapy and its implication for cardiac events in cardiac sarcoidosis and primarily preserved left ventricular ejection fraction. J Nucl Cardiol 2019. https://doi.org/10.1007/s12350-01901916-4.

8. Muser D, Santangeli P, Castro SA, Liang JJ, Enriquez A, Werner $\mathrm{TJ}$, et al. Prognostic role of serial quantitative evaluation of (18)F- fluorodeoxyglucose uptake by PET/CT in patients with cardiac sarcoidosis presenting with ventricular tachycardia. Eur J Nucl Med Mol Imaging 2018;45:1394-404.

9. Osborne MT, Hulten EA, Singh A, Waller AH, Bittencourt MS, Stewart GC, et al. Reduction in (1)(8)F-fluorodeoxyglucose uptake on serial cardiac positron emission tomography is associated with improved left ventricular ejection fraction in patients with cardiac sarcoidosis. J Nucl Cardiol 2014;21:166-74.

10. Ahmadian A, Brogan A, Berman J, Sverdlov AL, Mercier G, Mazzini M, et al. Quantitative interpretation of FDG PET/CT with myocardial perfusion imaging increases diagnostic information in the evaluation of cardiac sarcoidosis. J Nucl Cardiol 2014;21:92539.

11. Manabe O, Kroenke M, Aikawa T, Murayama A, Naya M, Masuda A, et al. Volume-based glucose metabolic analysis of FDG PET/ CT: The optimum threshold and conditions to suppress physiological myocardial uptake. J Nucl Cardiol 2019;26:909-18.

12. Bateman TM. Cardiac sarcoidosis: An important niche for PET, but a journey just begun. J Nucl Cardiol 2017;24:425-8.

13. Sperry BW, Tamarappoo BK, Oldan JD, Javed O, Culver DA, Brunken R, et al. Prognostic impact of extent, severity, and heterogeneity of abnormalities on (18)F-FDG PET scans for suspected cardiac sarcoidosis. JACC Cardiovasc Imaging 2018;11:336-45.

14. Vontobel J, Liga R, Possner M, Clerc OF, Mikulicic F, VeitHaibach $\mathrm{P}$, et al. MR-based attenuation correction for cardiac FDG PET on a hybrid PET/MRI scanner: Comparison with standard CT attenuation correction. Eur $\mathrm{J}$ Nucl Med Mol Imaging 2015;42:1574-80

15. Wicks EC, Menezes LJ, Barnes A, Mohiddin SA, Sekhri N, Porter JC, et al. Diagnostic accuracy and prognostic value of simultaneous hybrid 18F-fluorodeoxyglucose positron emission tomography/magnetic resonance imaging in cardiac sarcoidosis. Eur Heart J Cardiovasc Imaging 2018;19:757-67.

Publisher's Note Springer Nature remains neutral with regard to jurisdictional claims in published maps and institutional affiliations. 